PHYSICAL REVIEW D 91, 021302(R) (2015)

\title{
New Higgs inflation in a no-scale supersymmetric SU(5) GUT
}

\author{
John Ellis, ${ }^{1, *}$ Hong-Jian He, ${ }^{2, \dagger}$ and Zhong-Zhi Xianyu ${ }^{2,+}$ \\ ${ }^{1}$ Theoretical Particle Physics and Cosmology Group, Department of Physics, King's College London, \\ London WC2R 2LS, United Kingdom and Theory Division, CERN, CH-1211 Geneva 23, Switzerland \\ ${ }^{2}$ Institute of Modern Physics and Center for High Energy Physics, Tsinghua University, Beijing 100084, \\ China and Center for High Energy Physics, Peking University, Beijing 100871, China
}

(Received 27 November 2014; published 21 January 2015)

\begin{abstract}
Higgs inflation is attractive because it identifies inflaton with the electroweak Higgs boson. In this work, we construct a new class of supersymmetric Higgs inflationary models in no-scale supergravity with an SU(5) grand unified theory (GUT) group. Extending the no-scale Kähler potential and SU(5) GUT superpotential, we derive a generic potential for Higgs inflation that includes the quadratic monomial potential and a Starobinsky-type potential as special limits. This type of models can accommodate a wide range of the tensor-to-scalar ratio $r=\mathcal{O}\left(10^{-3}-10^{-1}\right)$ as well as a scalar spectral index $n_{s} \sim 0.96$.

DOI: 10.1103/PhysRevD.91.021302

PACS numbers: 98.80.Cq, 04.65.+e, 12.10.-g, 12.60.Jv
\end{abstract}

\section{INTRODUCTION}

Cosmological inflation [1] resolves the conceptual dilemma of the standard big-bang cosmology such as the horizon and flatness problems, and predicts that large-scale structures in the Universe originated from a nearly scale-invariant spectrum of density perturbations, which is well consistent with cosmological observations $[2,3]$. Theories of cosmological inflation postulate a scalar field, the inflaton, whose field energy drove an early epoch of near-exponential expansion. Before the LHC Higgs discovery, it was very tempting to identify this inflaton as the Higgs boson of the standard model (SM) [4], a very economical and predictive scenario. However, the recent LHC and Tevatron measurements of the Higgs and top quark masses indicate that the SM Higgs potential turns negative at $\sim 10^{11} \mathrm{GeV}$ [5], which is lower than the typical cosmic inflation scale $\sim 10^{16} \mathrm{GeV}$. This means that new physics is indispensable for our Universe to reach a stable electroweak vacuum after inflation.

On the other hand, the SM suffers from the naturalness problem of stabilizing the electroweak scale against radiative corrections to Higgs mass, for which one possible solution is low-energy supersymmetry (SUSY). Remarkably, it was shown [6] that the instability of the SM Higgs potential can be cured without severe fine-tuning by adding bosonic and fermionic degrees of freedom, ending up with a theory much like SUSY. In the simplest example along this line, it was also shown [7] that adding a new boson-fermion pair does lead to successful Higgs inflation with a wider range of the

\footnotetext{
*John.Ellis@cern.ch

hjhe@tsinghua.edu.cn

‡xianyuzhongzhi@gmail.com
}

Published by the American Physical Society under the terms of the Creative Commons Attribution 3.0 License. Further distribution of this work must maintain attribution to the author(s) and the published article's title, journal citation, and DOI. tensor-to-scalar ratio than that of the conventional Higgs inflation.

Combining Higgs inflation with SUSY is a challenging task. For instance, it was found in [8] that building Higgs inflation in the minimal supersymmetric standard model (MSSM) with a minimal Kähler potential is not viable. On the other hand, in the next-to-minimal supersymmetric standard model (NMSSM) one encounters a tachyonic instability along the direction of the additional singlet scalar [9], though this can be cured by adding higher-order terms to the Kähler potential [10]. Models of this kind were built in the minimal SU(5) grand unified theory (GUT) with a (strongly) modified no-scale Kähler potential and invoking a large nonminimal Higgs-curvature coupling, which gave a small tensor-to-scalar ratio $r$ [11]. Another type of GUT inflation model is $F$-term hybrid inflation, which generally leads to very small $r$ as well [12], though an enhanced value of $r$ could be achieved with a particular choice of Kähler potential [13]. Finally, it was shown in [14] that no-scale supergravity naturally accommodates models of inflation that yield predictions similar to the Starobinsky model [15] and the original model of Higgs inflation [4], as well as allowing more general inflationary potentials that could yield larger values of $r$ [16].

In this work, we construct a new class of Higgs inflation models, using the framework of no-scale supergravity (SUGRA) [17] and embedded in the supersymmetric SU(5) GUT. There are many motivations for this construction, of which we mention three. First, the inflation scale may well be close to the GUT scale, according to cosmological microwave background (CMB) measurements $[2,3]$, so it is very attractive to embed Higgs inflation into a GUT. Second, no-scale SUGRA emerges from simple compactifications of string theory [18]. Third, the flat directions in no-scale SUGRA are advantageous for cosmological applications [19].

For the new type of supersymmetric Higgs inflation in the no-scale SU(5) GUT framework, we adopt the minimal field 
content, with simple extensions of the no-scale Kähler potential and minimal SU(5) GUT superpotential. We derive a generic inflationary potential that interpolates between a quadratic monomial potential and a Starobinsky-type potential. The corresponding predictions of the tensor-to-scalar ratio $r$ and spectral index $n_{s}$ can accommodate the Planck and BICEP2 observations [2,3]. A notable feature of this no-scale SUSY GUT Higgs inflation scenario is that it does not invoke any nonminimal coupling between the Higgs fields and the Ricci curvature; i.e., all Higgs bosons couple minimally to gravity via the energy-momentum tensor. This is an essential difference between our construction and the conventional SM Higgs inflation [4] as well as its previous SUSY and GUT extensions [8-11]. Finally, we will further analyze the stability of inflation trajectories in all directions of field space and demonstrate the consistency.

\section{NEW HIGGS INFLATION WITH NO-SCALE SUGRA}

Our Higgs inflation with no-scale SUGRA and SU(5) GUT contains the following chiral fields as ingredients: a singlet modulus field $T$ that may arise from string compactification, a GUT Higgs multiplet $\Sigma$ in the adjoint representation of $\mathrm{SU}(5)$, and a pair of Higgs multiplets $H_{1}$ and $H_{2}$ belonging to $\mathbf{5}$ and $\overline{\mathbf{5}}$ representations of SU(5), respectively. We postulate the following extended no-scale Kähler potential $K$, which is a Hermitian function of these superfields,

$$
\begin{aligned}
K= & -3 \log \left[T+T^{*}-\frac{1}{3} \operatorname{tr}\left(\Sigma^{\dagger} \Sigma\right)\right. \\
& \left.-\frac{1}{3}\left(\left|H_{1}\right|^{2}+\left|H_{2}\right|^{2}-\zeta\left(H_{1} H_{2}+\text { H.c. }\right)\right)\right],
\end{aligned}
$$

where we set the reduced Planck mass $M_{\mathrm{Pl}}=1$ as a mass unit, and $\zeta$ is a dimensionless parameter. The theory obeys a simple $\mathbb{Z}_{2}$ symmetry, under which $\Sigma$ is odd and all other fields are even. We also postulate the following holomorphic superpotential $W$,

$$
W=W_{\Sigma}+W_{H}
$$

where the $\Sigma$ part

$$
W_{\Sigma}=-\frac{1}{2} m \operatorname{tr}\left(\Sigma^{2}\right)+\frac{1}{4} \tilde{\lambda} \operatorname{tr}\left(\Sigma^{4}\right)
$$

ensures the correct GUT-breaking vacuum for the GUT Higgs $\Sigma$, and the $H$ part

$$
W_{H}=\mu H_{1} H_{2}-\tilde{\beta}_{1} H_{1} \Sigma^{2} H_{2}+\beta_{2}\left(H_{1} H_{2}\right)^{2}
$$

generates desired triplet-doublet splitting for the fundamental Higgs multiplets $H_{1}$ and $H_{2}$. Due to the $\mathbb{Z}_{2}$ symmetry, the trilinear terms $\operatorname{tr}\left(\Sigma^{3}\right)$ and $H_{1} \Sigma H_{2}$ are absent in $W_{\Sigma}$ and $W_{H}$, respectively.

When considering spontaneous breaking of the GUT gauge group $\mathrm{SU}(5)$ down to the $\mathrm{SM}$ gauge group $\mathrm{SU}(3) \otimes \mathrm{SU}(2) \otimes \mathrm{U}(1)$, the adjoint Higgs field $\Sigma$ contains the relevant component, $\Sigma \supset \sqrt{2 / 15} \operatorname{diag}(1,1,1,-3 / 2$, $-3 / 2) \chi$, where $\chi$ is a singlet chiral multiplet, in terms of which the superpotential $W_{\Sigma}$ reduces to

$$
W_{\Sigma}=-\frac{1}{2} m \chi^{2}+\frac{7}{120} \tilde{\lambda} \chi^{4}
$$

The $\chi$ field should have its vacuum expectation value (VEV) take a value $\langle\chi\rangle \equiv u \simeq 2 \times 10^{16} \mathrm{GeV}$, as determined by the SUSY GUT gauge unification. The stationary condition $\partial W / \partial \chi=0$ yields $m=\lambda u^{2}$, with $\lambda \equiv \frac{7}{30} \tilde{\lambda}$.

In the presence of the GUT-breaking VEV $u$, the Higgs multiplets $H_{1}$ and $H_{2}$ split into $H_{1}=\left(H_{c}, H_{u}\right)^{T}$ and $H_{2}=\left(\bar{H}_{c}, H_{d}\right)^{T}$ as usual, where $\left(H_{c}, \bar{H}_{c}\right)$ are color SU(3) triplets and $\left(H_{u}, H_{d}\right)$ are weak $\mathrm{SU}(2)$ doublets. Thus, the $H$ part of the superpotential becomes

$$
\begin{aligned}
W_{H}= & H_{c}\left(\mu-\frac{4}{9} \beta_{1} \chi^{2}\right) \bar{H}_{c}+H_{u}\left(\mu-\beta_{1} \chi^{2}\right) H_{d} \\
& +\beta_{2}\left(H_{c} \bar{H}_{c}+H_{u} H_{d}\right)^{2},
\end{aligned}
$$

where $\beta_{1} \equiv \frac{3}{10} \tilde{\beta}_{1}$. In order for the two Higgs doublets $\left(H_{u}, H_{d}\right)$ to remain light (at the weak scale) while the colored Higgs triplets $\left(H_{c}, \bar{H}_{c}\right)$ become heavy, we set $\mu \simeq \beta_{1} u^{2}$. The color-triplet Higgs bosons then acquire a large mass $M_{c}=\frac{5}{9} \beta_{1} u^{2}$ at tree level. We parametrize the Higgs doublets $\left(H_{u}, H_{d}\right)$ as $H_{u}=\left(H_{u}^{+}, H_{u}^{0}\right)^{T}$ and $H_{d}=$ $\left(H_{d}^{0}, H_{d}^{-}\right)^{T}$, and identify a linear combination of $H_{u}^{0}$ and $H_{d}^{0}$ as the inflaton. The colored components $\left(H_{c}, \bar{H}_{c}\right)$ and the electrically charged components $\left(H_{u}^{+}, H_{d}^{-}\right)$do not play important roles during inflation, as we will discuss in Sec. IV.

Examples of modulus stabilization were studied in [14], and we assume here that nonperturbative ultraviolet dynamics fixes the VEV of the modulus field to be $T=T^{*}=1 / 2$ [20]. After these simplifications, the Kähler potential and the superpotential become

$$
\begin{aligned}
K= & -3 \log \left[1-\frac{1}{3}\left(|\chi|^{2}+\left|H_{u}^{0}\right|^{2}+\left|H_{d}^{0}\right|^{2}\right.\right. \\
& \left.\left.-\zeta\left(H_{u}^{0} H_{d}^{0}+\text { H.c. }\right)\right)\right] \\
W= & \beta_{1} H_{u}^{0}\left(u^{2}-\chi^{2}\right) H_{d}^{0}+\beta_{2}\left(H_{u}^{0} H_{d}^{0}\right)^{2} \\
& -\frac{1}{2} \lambda u^{2} \chi^{2}+\frac{1}{4} \lambda \chi^{4} .
\end{aligned}
$$


NEW HIGGS INFLATION IN A NO-SCALE ...

This is the basis for our following analysis of Higgs inflation in the no-scale SUSY SU(5) GUT.

\section{HIGGS INFLATION POTENTIAL AND OBSERVABLES}

The $F$-term scalar potential $V$ takes the following standard form,

$$
V=e^{G}\left(K_{i j^{*}} \frac{\partial G}{\partial \phi_{i}} \frac{\partial G}{\partial \phi_{j}^{*}}-3\right),
$$

where $G=K+\log W+\log W^{*}, K_{i j^{*}}$ is the inverse of Kähler metric $K^{i j^{*}} \equiv \partial^{2} K / \partial \phi_{i} \partial \phi_{j}^{*}$, and $\phi_{i}$ denotes generic scalar fields. We parametrize the neutral scalars explicitly as $H_{u}^{0}=\frac{1}{2}(X+Y) e^{\mathrm{i} \theta}$ and $H_{d}^{0}=\frac{1}{2}(X-Y) e^{\mathrm{i} \varphi}$, which give

$$
X=\left|H_{u}^{0}\right|+\left|H_{d}^{0}\right|, \quad Y=\left|H_{u}^{0}\right|-\left|H_{d}^{0}\right| .
$$

The $D$-flatness condition sets $Y=0$, and we further take $\theta=\varphi=0$ in the following (the validity of this choice will be examined in Sec. IV). Thus, we can identify the Higgs combination, $X=\left|H_{u}^{0}\right|+\left|H_{d}^{0}\right|$, as the inflaton. Moreover, during inflation the minimum of the $\chi$ field for a given $X$ is always at $\chi=0$, as will be examined in Sec. IV, so we also set $\chi=0$ from now on. Thus, the scalar potential $V$ becomes a function of $X$ alone, and takes the form

$$
V(X)=\frac{\beta_{1}^{2} u^{4}\left(1-\frac{\beta_{2}}{2 \beta_{1} u^{2}} X^{2}\right)^{2} X^{2}}{2\left(1-\frac{1-\zeta}{6} X^{2}\right)^{2}},
$$

which is the basis for our subsequent analysis.

The potential (10) displays a singularity at $X^{2}=6 /$ $(1-\zeta)$. The presence of such a singularity is ubiquitous in no-scale supergravity due to the form of the Kähler potential. It would lead to an exponentially steep potential in terms of a canonically normalized scalar field, violating the slow-roll condition. To cure this singularity, we impose the relation $\beta_{2}=\frac{1}{3} \beta_{1}(1-\zeta) u^{2}$, in which case the scalar potential simplifies to a quadratic monomial,

$$
V(X)=\frac{1}{2} \beta_{1}^{2} u^{4} X^{2}
$$

This does not lead to a quadratic chaotic inflation model, however, because the inflaton field $X$ is not canonically normalized, and its kinetic term takes the following form

$$
\mathcal{L}_{K}(X)=\frac{1-\frac{\zeta(1-\zeta)}{6} X^{2}}{2\left(1-\frac{1-\zeta}{6} X^{2}\right)^{2}}\left(\partial_{\mu} X\right)^{2} .
$$

Thus, we derive the canonically normalized inflaton field $h$ as a function of $X$,
PHYSICAL REVIEW D 91, 021302(R) (2015)

$$
\begin{aligned}
h= & \sqrt{6} \operatorname{arctanh} \frac{(1-\zeta) X}{\sqrt{6\left(1-\frac{1}{6} \zeta(1-\zeta) X^{2}\right)}} \\
& -\sqrt{\frac{6 \zeta}{1-\zeta}} \arcsin \left(\sqrt{\left.\frac{\zeta(1-\zeta)}{6} X\right) .}\right.
\end{aligned}
$$

From Eq. (13), we recognize two interesting limiting cases, $\zeta=0$ and $\zeta=1$, which can be worked out analytically.

When $\zeta=0$, we have the simplified relation $X(h)=$ $\sqrt{6} \tanh (h / \sqrt{6})$, and the potential $V(h)$ becomes

$$
V(h)=3 \beta_{1}^{2} u^{4} \tanh ^{2} \frac{h}{\sqrt{6}},
$$

which is exponentially flat at large $h$, and analogous to the Starobinsky model [15]. In this case, we can derive the following predictions for the primary inflationary observables, $\left(n_{s}, r\right) \simeq(0.967,0.003)$ for $60 e$-foldings, which are similar to the predictions of the Starobinsky model [15] and conventional Higgs inflation [4], as expected.

On the other hand, when $\zeta=1$, the $X$ field is already normalized canonically, $X(h)=h$, and we recover a quadratic monomial potential. This limit therefore yields the same predictions as quadratic chaotic inflation, namely, $\left(n_{s}, r\right) \simeq(0.967,0.130)$ for $60 e$-foldings.

When $\zeta$ varies between 0 and 1 , we obtain a class of inflation potentials that extrapolate between the quadratic monomial and Starobinsky-type potentials. The predictions for $\left(n_{s}, r\right)$ can be worked out numerically. We plot them in Fig. 1(a), where the green (yellow) dots and their attached curves represent predictions with 50(60) $e$-foldings. In this plot, the round (square) dots correspond to $\zeta=0(\zeta=1)$. The horizontal strips attached to the lower round dots correspond to the effect of varying $\zeta \in[0,0.1]$ (from right to left), while the upper strips attached to the square dots depict the effect of varying $\zeta \in[0.9,1]$ (from left to right). For comparison, we have depicted the recent observational limits $[2,3]$ as the shaded red and blue (pink and light-blue) contours at the $68 \%(95 \%)$ confidence level. We see that our predictions for $\left(n_{s}, r\right)$ are well compatible with the current data.

For Fig. 1(a), we imposed the constraint $\beta_{2}=$ $\frac{1}{3} \beta_{1}(1-\zeta) u^{2}$, and it is interesting to check what happens when $\beta_{2}$ deviates slightly from this relation. For this purpose, our starting point will be (10) and (13). We find that shifting $\beta_{2}$ according to $\beta_{2}=\frac{1}{3} \beta_{1}(1-\zeta+\delta) u^{2}$, with $\delta$ varying over the range $\pm\left(1.2 \times 10^{-3}\right)$, will make the predicted $\left(n_{s}, r\right)$ values vary as shown by the green and yellow strips (attached to the corresponding dots) in Fig. 1(b). Here the three pairs of round dots from top to bottom correspond to $\zeta=(1,0.98,0.95)$, while the green (yellow) dots and strips represent the predictions with 50 (60) $e$-foldings. Figure 1(b) shows again that the predicted values of $\left(n_{s}, r\right)$ agree well with the current experimental limits $[2,3]$. Together with the minimal case (11) presented in Fig. 1(a), Fig. 1(b) shows that our model predicts the 

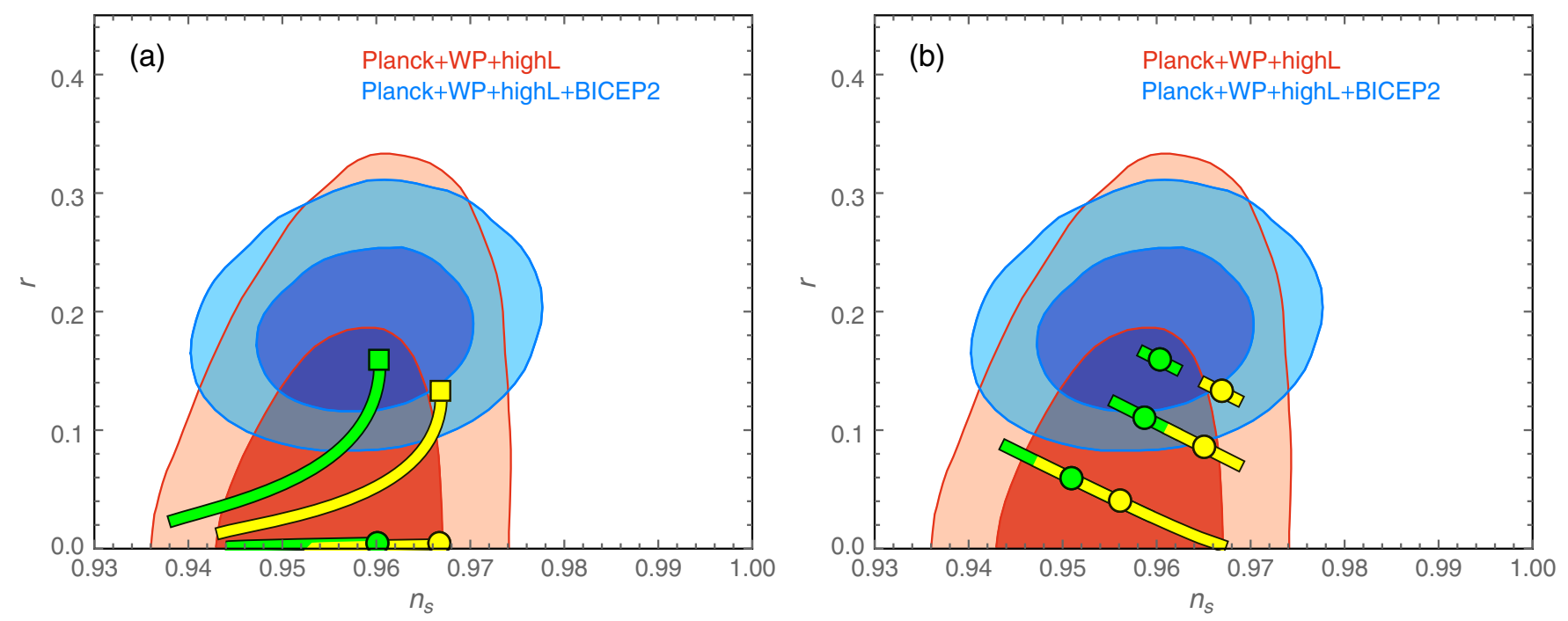

FIG. 1 (color online). The predictions of our no-scale SUSY GUT model of Higgs inflation for the spectral index $n_{s}$ and tensor-toscalar ratio $r$. The green (yellow) dots and curves represent predictions with 50(60) $e$-foldings. In plot (a), the condition $\beta_{2}=\frac{1}{3} \beta_{1}(1-\zeta) u^{2}$ is imposed and the round (square) dots correspond to $\zeta=0(\zeta=1)$. The horizontal strips attached to the lower round dots correspond to the effect of varying $\zeta \in[0,0.1]$ (from right to left), while the upper strips attached to the square dots depict the effect of varying $\zeta \in[0.9,1]$ (from left to right). In plot (b), the three pairs of dots from top to bottom correspond to $\zeta=(1,0.98,0.95)$ and $\delta=0$. The strip attached to each round dot describes the effect of varying $\delta$ over the range $\pm\left(1.2 \times 10^{-3}\right)$, via the shifted relation $\beta_{2}=\frac{1}{3} \beta_{1}(1-\zeta+\delta) u^{2}$. In each plot, the shaded red and blue (pink and light-blue) contours represent the observational limits at the $68 \%(95 \%)$ confidence level as given by Ref. [3].

range of the tensor-to-scalar ratio $r$ to be in the range $\mathcal{O}\left(10^{-3}-10^{-1}\right)$, without contrived theoretical inputs [21].

The coefficient $\beta_{1}$ in the potential (11) is fixed by the amplitude of scalar perturbations, $(V / \epsilon)^{1 / 4} \simeq 0.027$, as measured by the Planck satellite [2]. This yields $\beta_{1} \simeq$ 0.06 with little variation when $\zeta$ changes between 0 and 1 . Taking the GUT-breaking scale $u \simeq 0.01$ in units of the reduced Planck mass $M_{\mathrm{Pl}} \simeq 2.44 \times 10^{18} \mathrm{GeV}$, we estimate the mass of colored-triplet Higgs bosons to be $M_{c} \simeq$ $0.8 \times 10^{13} \mathrm{GeV}$. There are arguments [23] from proton stability and gauge coupling unification that prefer $M_{c}$ to be much larger than $10^{13} \mathrm{GeV}$ [24]. However, the proton stability argument relies on particular hypotheses about the mechanism for generating the light fermion masses and mixing in the SU(5) GUT. This is a known problem of the model and can be evaded in various ways (which are beyond the scope of this short paper). Also, the unification argument depends on oversimplified assumptions about physics around and beyond the GUT scale that may be relaxed without affecting our main predictions. For example, the nonminimal contributions to the gauge kinetic function in supergravity will modify the gauge unification condition.

Finally, we note that the $\beta_{2}$-term in the superpotential (6) induces a new dimension-4 term (involving the light Higgs doublets $H_{u}$ and $H_{d}$ ) in the Higgs potential. However, the coefficient of this term is only of the order of $\bar{\mu} \beta_{2} \simeq \frac{1}{3} \beta_{1}(1-$ $\zeta) u^{2} \bar{\mu}$ in Planck-mass units [or $\bar{\mu} \beta_{2} \simeq 0.02(1-\zeta) u^{2} \bar{\mu}$ / $M_{\mathrm{Pl}}^{3} \lll 1$ with the Planck-mass dependence exhibited], where $\bar{\mu}=\mu-\beta_{1} u^{2}=\mathcal{O}(\mathrm{TeV})$ is the residual $\mu$-term at the electroweak scale. Hence, this new term is negligible for low-energy SUSY phenomenology. Other induced higherdimensional operators in the Higgs potential are even more suppressed by powers of $1 / M_{\mathrm{Pl}}$.

\section{STABILITY OF THE INFLATIONARY TRAJECTORY}

In this section, we study the stability of inflationary trajectory in our Higgs inflation scenario. Checking stability is a necessary and nontrivial task since some previous proposals for SUSY Higgs inflation suffer from tachyonic instabilities, as mentioned in Sec. I. For this purpose, we compute the effective mass matrix $M_{i j} \equiv \frac{1}{2}\left(\partial^{2} V / \partial \phi_{i} \partial \phi_{j}\right)$ in all scalar directions $\phi_{i}$ along the inflationary trajectory for $X<\sqrt{6 /(1-\zeta)}$ (with $\zeta<1$ ), and all other fields $\phi_{j}=0$. The mass matrix is block diagonal, and the analysis can be subdivided into four independent sectors, namely, the colored Higgs fields $\left(H_{c}, \bar{H}_{c}\right)$, the charged Higgs fields $\left(H_{u}^{ \pm}, H_{d}^{ \pm}\right)$, the phases of neutral Higgs fields $(\theta, \varphi)$, and the two components $(s, t)$ of the SM gauge-singlet scalar $\chi=s+i t$.

In the case of the color-triplet Higgs fields $\left(H_{c}, \bar{H}_{c}\right)$, we consider the first color component of each field for simplicity, which we parametrize as $h_{c} e^{\mathrm{i} \theta_{c}}$ and $\bar{h}_{c} e^{\mathrm{i} \bar{\theta}_{c}}$. Steepness along the $h_{c}-\bar{h}_{c}$ direction is guaranteed by the $D$-term in the effective potential, and the effective mass along the $h_{c}+\bar{h}_{c}$ direction is $M_{c c}^{2}=2 \beta_{1}^{2} u^{4}$ [25]. We have 
NEW HIGGS INFLATION IN A NO-SCALE ...

also checked that there are no instabilities in the $\left(\theta_{c}, \bar{\theta}_{c}\right)$ directions. Therefore, there is no instability in this sector. The check for the charged Higgs sector is similar.

Regarding the angular parts of neutral Higgs bosons $(\theta, \varphi)$, we find the following elements in the $2 \times 2$ effective mass matrix $\mathcal{M}^{2}$,

$$
\begin{aligned}
M_{\theta \theta}^{2}=M_{\varphi \varphi}^{2}= & \frac{\beta_{1}^{2} u^{4} X^{4}}{\left(1-\frac{1-\zeta}{6} X^{2}\right)^{4}}\left[\frac{1}{3}+\frac{8 \zeta^{2}+17 \zeta-7}{144} X^{2}\right. \\
& \left.+\frac{5 \zeta^{3}-6 \zeta+1}{432} X^{4}+\frac{(1-\zeta)^{2}\left(17 \zeta^{2}+4 \zeta-3\right)}{20736} X^{6}\right],
\end{aligned}
$$

$$
\begin{aligned}
M_{\theta \varphi}^{2}=M_{\varphi \theta}^{2} & =\frac{\beta_{1}^{2} u^{4} X^{4}}{\left(1-\frac{1-\zeta}{6} X^{2}\right)^{4}}\left[\frac{3 \zeta+1}{12}+\frac{14 \zeta^{2}+5 \zeta-1}{144} X^{2}\right. \\
& \left.+\frac{13 \zeta^{3}-9 \zeta^{2}-3 \zeta-1}{864} X^{4}+\frac{\zeta(10 \zeta-1)(\zeta-1)^{2}}{10368} X^{6}\right] .
\end{aligned}
$$

The two eigenvalues of $\mathcal{M}^{2}$ are

$$
\begin{aligned}
M_{1}^{2}= & \frac{\beta_{1}^{2} u^{4} X^{4}}{\left(1-\frac{1-\chi}{6} X^{2}\right)^{4}}\left[\frac{3 \zeta+5}{12}+\frac{11 \zeta^{2}+11 \zeta-4}{72} X^{2}\right. \\
& +\frac{23 \zeta^{3}-9 \zeta^{2}-15 \zeta+1}{864} X^{4} \\
& \left.+\frac{(1-\zeta)^{2}\left(37 \zeta^{2}+2 \zeta-3\right)}{20736} X^{6}\right], \\
M_{2}^{2}= & \frac{\beta_{1}^{2} u^{4} X^{4}}{\left(1-\frac{1-\zeta}{6} X^{2}\right)^{4}}\left[\frac{1-\zeta}{4}-\frac{(1-\zeta)^{2}}{24} X^{2}\right. \\
& \left.+\frac{(1-\zeta)^{3}}{288} X^{4}-\frac{(1-\zeta)^{4}}{6912} X^{6}\right],
\end{aligned}
$$

which are both positive for $\zeta \in[0,1]$ and $X<\sqrt{6 /(1-\zeta)}$, during the inflationary epoch. Hence, the effective mass matrix $\mathcal{M}^{2}$ is positive definite along the inflation trajectory.

Finally, we compute the effective mass matrix for the real and imaginary parts of the singlet field $\chi$, up to corrections of $\mathcal{O}\left(u^{2}\right)$,
PHYSICAL REVIEW D 91, 021302(R) (2015)

$$
\begin{aligned}
& M_{s s}^{2}=M_{t t}^{2}=\frac{\beta_{1}^{2} X^{4}}{4\left(1-\frac{1-\zeta}{6} X^{2}\right)^{2}}, \\
& M_{s t}^{2}=0 .
\end{aligned}
$$

This is always positive definite, so the $(s, t)$ directions are also stable. In summary, we have systematically verified that the inflationary trajectory is stable in all scalar directions for typical parameter choices.

\section{CONCLUSIONS}

Higgs inflation provides a highly economical and predictive approach for the cosmic inflationary paradigm. In this work, we have proposed a new class of Higgs inflation models in the framework of an SU(5) GUT embedded in no-scale supergravity. The structure of this type of models is fairly simple, since it includes a nearminimal no-scale Kähler potential and simple superpotential with terms up to fourth order in the Higgs chiral multiplets. The resultant inflaton potential has a variable form, capable of interpolating between quadratic monomial and Starobinsky-type potentials. These models can therefore accommodate a wide range of values of the tensor-to-scalar ratio $r$, while predicting values of the scalar spectral index $n_{s}$ that are compatible with the present experimental limits. Future CMB observations will soon measure or constrain more precisely the possible value of $r$. These will further test the predictions of this new class of Higgs inflation models with no-scale SUSY GUT.

\section{ACKNOWLEDGMENTS}

The work of J.E. was supported in part by the London Centre for Terauniverse Studies (LCTS), using funding from the European Research Council via the Advanced Investigator Grant No. 267352, and in part by the STFC Grant No. ST/J002798/1. H.-J. H. and Z.-Z. X. were supported by the Chinese NSF (under Grants No. 11275101 and No. 11135003) and the National Basic Research Program (Grant No. 2010CB833000).
[1] For reviews, see A. D. Linde, Particle Physics and Inflationary Cosmology (Harwood, Chur, Switzerland, 1990); K. A. Olive, Phys. Rep. 190, 307 (1990); D. H. Lyth and A. Riotto, Phys. Rep. 314, 1 (1999); J. Martin, C. Ringeval, and V. Vennin, Phys. Dark Univ. 5-6, 75 (2014), and references therein.
[2] P. A. R. Ade et al. (Planck Collaboration), Astron. Astrophys. 571, A22 (2014).

[3] P. A. R. Ade et al. (BICEP2 Collaboration), Phys. Rev. Lett. 112, 241101 (2014).

[4] F. L. Bezrukov and M. Shaposhnikov, Phys. Lett. B 659, 703 (2008); for a recent review, see F. Bezrukov, Classical 
Quantum Gravity 30, 214001 (2013), and references therein.

[5] J. R. Espinosa, G. Giudice, and A. Riotto, J. Cosmol. Astropart. Phys. 05 (2008) 002; J. Ellis, J. R. Espinosa, G. F. Giudice, A. Hoecker, and A. Riotto, Phys. Lett. B 679, 369 (2009); D. Buttazzo, G. Degrassi, P. Paolo Giardino, G. F. Giudice, F. Sala, A. Salvio, and A. Strumia, J. High Energy Phys. 12 (2013) 089; M. Fairbairn and R. Hogan, Phys. Rev. Lett. 112, 201801 (2014); A. Kobakhidze and A. Spencer-Smith, arXiv:1404.4709; A. Hook, J. Kearney, B. Shakya, and K. M. Zurek, arXiv:1404.5953; A. SpencerSmith, arXiv:1405.1975, and references therein.

[6] J. Ellis and D. Ross, Phys. Lett. B 506, 331 (2001).

[7] H. J. He and Z. Z. Xianyu, J. Cosmol. Astropart. Phys. 10 (2014) 019.

[8] M. B. Einhorn and D. R. T. Jones, J. High Energy Phys. 03 (2010) 026.

[9] S. Ferrara, R. Kallosh, A. Linde, A. Marrani, and A. V. Proeyen, Phys. Rev. D 82, 045003 (2010).

[10] S. Ferrara, R. Kallosh, A. Linde, A. Marrani, and A. V. Proeyen, Phys. Rev. D 83, 025008 (2011).

[11] M. Arai, S. Kawai, and N. Okada, Phys. Rev. D 84, 123515 (2011).

[12] G. Dvali, Q. Shafi, and R. K. Schaefer, Phys. Rev. Lett. 73, 1886 (1994); M. Civiletti, C. Pallis, and Q. Shafi, Phys. Lett. B 733, 276 (2014).

[13] F. Brummer, V. Domcke, and V. Sanz, J. Cosmol. Astropart. Phys. 08 (2014) 066.

[14] J. Ellis, D. V. Nanopoulos, and K. A. Olive, Phys. Rev. Lett. 111, 111301 (2013); J. Cosmol. Astropart. Phys. 10 (2013) 009.
[15] A. A. Starobinsky, Phys. Lett. 91B, 99 (1980); V. F. Mukhanov and G. V. Chibisov, Pis'ma Zh. Eksp. Teor. Fiz. 33, 549 (1981) [JETP Lett. 33, 532 (1981)].

[16] J. Ellis, M. A. G. Garcia, D. V. Nanopoulos, and K. A. Olive, J. Cosmol. Astropart. Phys. 05 (2014) 037; 08 (2014) 044; arXiv:1409.8197.

[17] J. Ellis, C. Kounnas, and D. V. Nanopoulos, Nucl. Phys. B247, 373 (1984).

[18] E. Witten, Phys. Lett. 155B, 151 (1985).

[19] A. Lahanas and D. V. Nanopoulos, Phys. Rep. 145, 1 (1987), and references therein.

[20] For discussions of moduli stabilization in the string framework, see M. Cicoli, S. de Alwis, and A. Westphal, J. High Energy Phys. 10 (2013) 199, and references therein.

[21] We do not discuss here two- or multifield effects [22], which would tend to suppress $r$.

[22] S. Kawai and J. Kim, arXiv:1411.5188, and references therein.

[23] E.g., T. Goto and T. Nihei, Phys. Rev. D 59, 115009 (1999); H. Murayama and A. Pierce, Phys. Rev. D 65, 055009 (2002).

[24] If, instead of the last two quartic terms in the superpotential $W_{H}$ of (4), we had used a more conventional trilinear term $\epsilon H_{1} \Sigma H_{2}$, the amplitude of scalar perturbations would have required $\epsilon \sim 10^{-6}$, which would have implied $M_{c} \simeq 10^{10} \mathrm{GeV}$. Avoiding such a rather low mass $M_{c}$ was in fact our principal motivation for imposing the $\mathbb{Z}_{2}$ symmetry discussed earlier.

[25] The effective mass $M_{c c}$ calculated here should not be confused with the color-triplet Higgs mass $M_{c}$ computed earlier. The mass $M_{c}$ was derived in the GUT-breaking vacuum, whereas $M_{c c}$ is evaluated at $\chi=0$, and is valid for $X \sim \mathcal{O}(1)$. 